\title{
Impact of removable partial dental prostheses on the oral health- related quality of life of a South African cohort with varied distributions of missing posterior teeth
}

\author{
Sadika Khan, Usuf Chikte and Ridwaan Omar
}

\begin{abstract}
Purpose: To determine the impact of removable partial dental prostheses on satisfying the daily functioning and quality of life (QoL) of adult patients with different distributions of missing posterior teeth.

Materials and Methods: A cross-sectional interventional study was carried out on 80 patients having variously distributed posteriorly shortened and interrupted arches. Treatment comprised provision of partial dentures by senior dental students, supervised by senior clinical teachers who had knowledge of the potential benefits of the shortened dental arch (SDA) concept. The Oral Impacts on Daily Performance Index was completed before and 6 months after prosthetic treatment across groups comprising Kennedy Classes I, II, and III arches. Analysis included descriptive statistics and associations and comparisons between variables.

Results: Mean age of patients was 57.4 years $(\mathrm{SD}=13.1)$, many were retired $(72.2 \%)$, and a majority were females (60\%). Most patients lived in urban areas (95\%), and were largely unemployed (63.3\%). At pretreatment, only $31.3 \%$ of patients reported having good dental health and satisfaction with their current oral state, while $82.5 \%$ said they had a great need for treatment. The negative oral impacts that were most frequently experienced were those of eating (67.5\%), smiling (50\%), and being emotionally disturbed (63.8\%). Posttreatment, $76.3 \%$ indicated good oral health and satisfaction with no significant differences between the 3 Kennedy groups. Any further negative impacts were reported mostly for Kennedy Classes I and II.

Conclusions: Overall, significant reductions of negative impacts were observed following treatment with dentures, across the 3 Kennedy groups, with respect to improved function, satisfaction, and oral health-related QoL. The findings confirm the reliance by partially dentate patients in all 3 Kennedy groups on dentures for improved oral health, although the possible benefits of the SDA concept as an alternative treatment option was not specifically explored.
\end{abstract}

Evidence from several sources recommends that reduced or interrupted dentitions should be categorized according to their ability to ensure satisfactory oral function. ${ }^{1-4}$ Studies on oral function suggest that oral health related quality of life (OHRQoL) can be related to 
the presence of nine or more pairs of anterior and posterior occluding teeth, $1,2,5$ and that anything less than this negatively affects patient satisfaction and OHRQoL. $1,2,5-7$ Generalizability of these results cannot be assumed, as contexts differ considerably regarding cultural and socioeconomic circumstances, which in turn have been shown to impact OHRQoL and patient satisfaction.5,7

Normative and perceived needs regarding the functional adequacy of partial edentulism, including reduced posterior dentitions, differ, ${ }^{2,8,9}$ and thus assessments for prosthetic replacement vary widely. In general, normative assessments of treatment needs, especially in older, partially dentate adults, exceed the perceived needs of the patients themselves. ${ }^{2,9}$ There is growing evidence that the prosthetic management approach, especially in such an older group of patients, should include treatment options predicated on the maintenance of a functional dentition.3,10-13 This differs from the traditional approach of a morphologically intact dentition being considered the determinant of satisfactory function.

The shortened dental arch (SDA) concept, introduced by Käyser in the 1980s, has been proposed as an alternative treatment option for older, partially dentate adults.1,3,4,10-26 The concept is functionally oriented and has been shown to satisfy the functional needs and OHRQoL of such patients in several population groups. ${ }^{11-33}$ The classic SDA is defined as having 20 occluding anterior and premolar teeth, although several variations relating to the number of posterior occluding pairs (POPs) of teeth have been described as well. 1,5,11-13,17-27 The benefits of the classic SDA and its many variations have been described in a global context $1,3,11,12,17-21,23-31$ and a South African context. $22,32,33$

Gotfredsen and Walls referred to the difficulties patients experience when expressing their satisfaction regarding their oral function, and advised that these patients should optimally be guided by clearly defined concepts and validated indicators when their needs are assessed and treatments recommended. ${ }^{1}$ Adopting a problem-orientented and patientcentered treatment approach would increase the possibility of achieving successful treatment outcomes. 34

Several statistically validated OHRQoL indicators are available that would simultaneously determine patients' clinical status and psychological and social dimensions when determining dental needs, that is, combining normative and perceived needs. ${ }^{2,35-39}$ The Oral Impacts on Daily Performance (OIDP) index, described by Adulyanon and Sheiham in 1997, has been used to assess diverse populations' dental needs and for planning dental services.36,39 Importantly, the OIDP adequately encompasses the concepts related to basic needs and demands. $2,36,39$

\section{http://repository.uwc.ac.za}


In studies conducted within the South African context, knowledge of the SDA among dentists in private practice and those teaching at a large dental institution was not widespread, and not surprisingly, it was rarely translated into clinical practice. ${ }^{22,32}$ The commonly accepted and applied method of treating such patients is with removable partial dental prostheses (RPDPs). Since patients tend to value and trust the judgements of clinicians without questioning the treatment offered (a clinician-centered approach), the impact and effect of treatment with RPDPs on patients' daily life, in light of alternatives such as the SDA approach, has not been adequately explored. In particular, no studies alluding to the functioning ability and OHRQoL benefits for patients with differing partially dentate scenarios as defined by their Kennedy classification, viz. Class I (which incorporates classic SDAs), II, and III, have been conducted in South Africa.

The aim of this study was to assess the impact of RPDPs on satisfying the functioning ability and OHRQoL of a group of partially dentate adult patients, with various distributions of missing posterior teeth according to Kennedy Class I, II, and III. The null hypothesis was formulated as follows: In partially dentate patients with a Kennedy Class I and II (posteriorly reduced) or Class III (discontinuous and interrupted) dental arch, the use of RPDPs do not influence daily functional ability, satisfaction, and OHRQoL.

\section{Materials and methods}

Ethical clearance (Registration No. 11/1/50 and S13/04/066) was obtained from the Research and Ethics Committees of the University of the Western Cape (UWC) and the Stellenbosch University. Written informed consent was obtained from the participants according to the Declaration of Helsinki.40 The study population for this cross-sectional interventional study comprised a convenience sample of partially dentate patients $(\mathrm{n}=$ 80), presenting to the clinic requesting replacement of missing posterior teeth with cobalt-chrome clasp-retained RPDPs. Patients had to have a Kennedy Class I, II (posterior reduced or shortened), or III (discontinuous or interrupted) dental arch and had to be considered suitable for treatment by senior dental students after a thorough screening by academic staff. After being fully informed about the nature and purpose of the study, and agreeing to participate, enrolled patients were interviewed by the principal researcher using the OIDP questionnaire prior to receiving any prosthetic treatment. Subsequent to the prosthetic treatment, and after the patients had worn the prosthesis for 6 months, the principal researcher again completed the OIDP questionnaire with patients so that they served as their own controls. Treatment comprised provision of patients with cobaltchrome clasp-retained RPDPs to replace all missing teeth by senior dental students supervised by clinical teachers.

The modified OIDP index (validated for the South African population) was used in this study and administered by the principal researcher.39 Patients' demographic details (age, gender, economic, and employment status) were recorded. Participants were classified into groups according to socioeconomic category (middle, low working class, no income) and occupation (professional, skilled, unskilled, and unemployed).39,41 In addition, responses to the

\section{http://repository.uwc.ac.za}


general and oral health questions were recorded using a 5-point Likert-type scale: for example, responses for rating aspects of dental health ranged from very poor (score of 1) to very good (score of 5) and for patient satisfaction from not at all satisfied (score of 1) to very satisfied (score of 5).40 With regard to the OIDP assessment, the sections that focused on the OHRQoL required a yes/no response for each of the 10 dimensions included, as well as for reasons for patients' particular responses. 39 The corresponding frequency and severity for each dimension was recorded using a 5-point Likert-type scale (no effect to very severe effect).39 Similarly for health behaviors (including dietary intake) and dental care habits, responses were again recorded using a yes/no response or a Likert-type scale.39

Frequencies were calculated for the demographic data and for oral impacts and oral health behaviors at pre- and post-intervention stages and recorded according to the first 3 Kennedy classifications. The associations between qualitative variables (e.g., dental health and need for dental treatment and oral impacts) were studied by drawing up contingency tables and applying the Chi-square test or Fisher's exact test where necessary ( $p$-values indicating the significance) at both pre- and post-intervention stages. For comparisons of means, the $t$-test or, when appropriate, the paired $t$-test was used. Cross tabulations were also completed between pre- and post-intervention responses using McNemar's test to observe statistically significant differences stated by the $p$-values. The data were analyzed using the Epi-Info and R-statistical programmes.

Table 1 Demographic distributions and postintervention patient responses according to Kennedy Classifications

\begin{tabular}{|c|c|c|c|c|}
\hline & Kennedy Class I & Kennedy Class II & Kennedy Class III & Total \\
\hline \multicolumn{5}{|l|}{ 1. Demographic data } \\
\hline Gender: Male & $13(35.1 \%)$ & $7(43.7 \%)$ & $12(44.4 \%)$ & $32(40 \%)$ \\
\hline Female & $24(64.9 \%)$ & $9(56.3 \%)$ & $15(55.6 \%)$ & $48(60 \%)$ \\
\hline \multirow[t]{2}{*}{ Age category } & $35-54: N=14$ & $29-54: N=5$ & $28-54: N=12$ & $31(38.8 \%)$ \\
\hline & $55-86: N=23$ & $55-79: \mathrm{N}=11$ & 55-83: $N=15$ & $49(61.2 \%)$ \\
\hline Location: Urban & 35 & 16 & 25 & $76(95 \%)$ \\
\hline 2. Sample size (N) & $\mathrm{N}=37$ & $N=16$ & $N=27$ & 80 \\
\hline \multicolumn{5}{|c|}{ Post-intervention } \\
\hline \multicolumn{5}{|l|}{ 3. Oral health: } \\
\hline Good dental health & $27(72 \%)$ & $12(75 \%)$ & $22(82 \%)$ & $76.3 \%$ \\
\hline Patients satisfied & $26(70 \%)$ & $12(75 \%)$ & $23(85 \%)$ & $76.3 \%$ \\
\hline Need treatment & $9(24.3 \%)$ & $3(18.7 \%)$ & $4(14.8 \%)$ & $20 \%$ \\
\hline \multicolumn{5}{|l|}{ 4. Negative oral impacts: } \\
\hline Eating & $9(24.3 \%)$ & $3(18.8 \%)$ & $4(14.8 \%)$ & $20 \%$ \\
\hline Smiling & $6(16.2 \%)$ & 0 & $3(11 \%)$ & $11.25 \%$ \\
\hline Emotional & $6(16.2 \%)$ & $3(18.8 \%)$ & $3(11 \%)$ & $15 \%$ \\
\hline Contact with family & $4(10.8 \%)$ & 0 & $3(11 \%)$ & $8.8 \%$ \\
\hline
\end{tabular}

\section{Results}

\section{Demographics}

The age range of participants was 28 to 86 years (mean age $=57.4, \mathrm{SD}=13.1$ ) with a $60 \%$ female majority. Most patients lived in urban areas (95\%), very few were in the upper middle class group (1.3\%), and most were retired (72.2\%). The majority of patients were

\section{http://repository.uwc.ac.za}


unemployed (63.3\%), with equal numbers within the other categories at $6.3 \%$ in the skilled and unskilled groups.

The demographic variables that may be considered as confounders were patients' general health, socioeconomic status, level of education, and residential location. From an assessment of the data, however, no significant results with respect to possible confounders were noted. Notably, post-intervention, whereas complaints or negative impacts were reported among patients from different socioeconomic and education levels, the only demographic variable showing significant differences was gender. It was also noted that most complaints for the different impacts post-intervention were by men in the Kennedy Class I and III groups, even though women formed the majority of the sample (Table 1).

\section{General oral health}

For all patients attending the prosthetic clinic for the specific purpose of receiving a RPDP, institutional protocol required that all basic restorative and periodontal procedures had to be completed before these prostheses were provided. Pretreatment, 31.3\% of the total sample indicated their perceived dental health as good or very good compared to a post-treatment proportion of $76.3 \%(p<0.0001)$. Correspondingly, patient satisfaction with perceived oral health was recorded as $76.3 \%$ ( $p<0.0001) 6$ months after receiving the prostheses. At post-intervention, for both these oral health indicators, non-significant differences were recorded across the Kennedy classifications, although the numbers of those reporting being most satisfied was from the Kennedy Class III group (Table 1). Prior to receiving their RPDPs, 82.5\% ( $p<0.0001)$ of the total sample had felt they were in great need of dental treatment while this need for further treatment decreased substantially (with the greatest need noted for the Kennedy Class I group) after provision of the RPDP (Table 1). Cross tabulations completed for the total sample, however, showed highly significant differences between pre- and post-intervention responses as specified by the $p$-values obtained after applying McNemar's test. At post-intervention, significant gender differences were observed, with more men indicating poor satisfaction and a greater need for more treatment.

\section{Oral impacts}

Total OIDP score measures prevalence (proportion of subjects reporting one or more daily oral impact), extent (number of daily performances affected), and severity (more severe effect in one performance) of oral impacts on daily life. Even though the total OIDP score at the pre-intervention stage was fairly low (20.7\%), signifying good self-rated oral health status, some specific negative oral impacts (eating, smiling, being emotional, and contact with family) were experienced almost daily. Based on the 5-point scale of responses ("no effect" to "very severe effect"), the negative impacts were reported to have affected their daily life severely; however, following treatment with a RPDP and after 6 months of use, the total OIDP score was reduced to $5.9 \%$. The acquisition of a RPDP, which was still worn by this cohort of patients, thus seemingly improved their perceived dental health and subsequently had a positive effect on their quality of life and OHRQoL.

\section{http://repository.uwc.ac.za}


Table 2 Pre-intervention associations between general health and oral impacts indicating patients' "Yes" responses

\begin{tabular}{|c|c|c|c|c|c|c|}
\hline & 1 & 2 & 3 & 4 & 5 & $p$-Value \\
\hline \multicolumn{7}{|l|}{ Dental health } \\
\hline Eating & 100 & 62.5 & 68 & 68.2 & 33.3 & ${ }^{5} 0.312$ \\
\hline Smiling & 100 & 62.5 & 32 & 45.5 & 33.3 & 0.024 \\
\hline Being emotional & 100 & 83.3 & 52 & 54.5 & 0 & 0.005 \\
\hline Contact with family & 100 & 50 & 24 & 36.4 & 33.3 & 0.013 \\
\hline \multicolumn{7}{|l|}{ Patient satisfaction } \\
\hline Eating & 76.5 & 68.8 & 54.5 & 73.7 & 66.7 & ${ }^{\S} 0.617$ \\
\hline Smiling & 100 & 43.8 & 50 & 21.1 & 16.7 & $<0.001$ \\
\hline Being emotional & 82.4 & 87.5 & 63.6 & 42.1 & 16.7 & 0.002 \\
\hline Contact with family & 76.5 & 50 & 31.8 & 26.3 & 0 & 0.003 \\
\hline \multicolumn{7}{|c|}{ Need for dental treatment } \\
\hline Eating & & 60 & 66.7 & 69 & 67.6 & ${ }^{\S} 0.997$ \\
\hline Smiling & & 0 & 33.3 & 41.4 & 67.6 & 0.009 \\
\hline Being emotional & & 20 & 33.3 & 55.2 & 83.8 & 0.002 \\
\hline Contact with family & & 0 & 33.3 & 31 & 56.8 & 0.034 \\
\hline
\end{tabular}

KEY:

Dental health:(1) Very poor, (2) Poor, (3) Fair, (4) Good, (5) Very good

Patient satisfaction:(1) Not at all satisfied, (5) Very satisfied

Need for dental treatment:(1) Not at all, (5) A great deal

$\S$ : $p$-value indicates that the results were not significant.

The oral impacts of speaking, cleaning teeth, physical activity (both light and vigorous), sleeping, and relaxing were unaffected by patients' oral state, and thus are not reported. The oral impacts experienced most frequently by patients with shortened and/or interrupted posterior dental arches were those of eating (67.5\%), smiling (50\%), and being emotional (63.8\%). Statistically significant reductions in the prevalence of negative impacts were observed for eating (20\%), smiling (11.3\%), and being emotional (15\%) following treatment with clasp-retained RPDPs across all Kennedy groups $(p<0.0001)$.

At post-intervention, the negative oral impacts affecting OHRQoL were mostly reported from men and from the Kennedy I and III groups for eating, smiling, and being emotional (Table 1). Only women reported negative oral impacts in the Kennedy Class II group for eating and being emotional. Most-negative OHRQoL impacts reported were in the following descending order: Kennedy Class I, Class III, and Class II groups, and for those impacts specified above (Table 1). Negative impacts were reported for patients from different age, socioeconomic, and occupation groups, but these were not significant. Only gender differences were significant (as mentioned previously).

\section{Associations between general oral health and oral impacts}

Preintervention stage: The association between negative oral impacts (eating, smiling, being emotional, and contact with family) and perceived dental health data were summarized in $5 \times 2$ cross tabulation frequency distributions (Table 2). While there was variation between the percentages, the differences between them for eating were not

\section{http://repository.uwc.ac.za}


statistically significant $\left(\chi^{2}=4.77 ; \mathrm{df}=4 ; p=0.312\right)$ according to the results of a Chisquare test. The responses of participants changed for eating (recorded as "no problems with eating") as their perceived dental health status improved (Table 1).

The results for smiling, being emotional, and contact with family versus perceived dental health are also recorded in Table 2. Here, the $p$-values indicate the significance, or otherwise, of association, and these were confirmed by Fischer's exact tests where needed. Patients' responses for dental health versus smiling showed a similar trend to the results for eating, but for smiling the trend was statistically significant $\left(\chi^{2}=11.26 ; \mathrm{df}=4 ; p\right.$ $=0.024)$. Similarly, for patient satisfaction, the trend was comparable to that of perceived dental health, with the percentage of respondents saying "Yes" (that is 'not satisfied') decreasing with improved dental health status; however, the trend was in the opposite direction with need for treatment versus the reported negative oral impacts (eating, smiling, being emotional, and contact with family); that is, the need for treatment was perceived as greater when patients indicated experiencing negative oral impacts.

\section{Discussion}

In this study, the oral impacts most noticeably affected preoperatively were eating, smiling, the emotional state of patients, and contact with family. Eating was possibly impacted by loss of posterior teeth and their different distributions, while concerns with smiling, given that all anterior teeth were present, may be attributed to missing premolar teeth, especially in patients with a broad smile.

Following treatment with RPDPs, patients generally expressed satisfaction as well as an improvement in oral impacts, oral functional satisfaction, and more specifically in OHRQoL, although differences across the three groups were noted. Overall OIDP scores were lower, indicating that the presence of a clasp-retained cobalt-chrome RPDP improved their selfrated oral health and also the importance that such a denture has for function, and possibly esthetics, among this cohort. Any negative responses reported after receiving RPDPs were from the Class I and III groups, and most were reported by the men. It is important to mention that the confounder, viz. provision of basic restorative and periodontal treatment prior to all such interventions, could have influenced the changes in their responses. The fact that the OIDP was completed 6 months after RPDP placement may, however, have reduced this potential effect.

The literature consistently states that the presence or absence of anterior teeth plays a major role in how patients respond to treatment with RPDPs, and thus to questionnaires or oral health indicators that focus on this treatment option. ${ }^{21}$ Having excluded such patients from our sample, the responses seem surprising in that a substantial number reported negative responses for smiling. Such a response might have been expected had a Kennedy Class IV group been included as a cohort. At the same time, it is known that many patients in the community from which our sample was drawn request to have their anterior teeth extracted as a culturally driven preference. Since the present focus was on reduced posterior arches,

\section{http://repository.uwc.ac.za}


these patients were deliberately excluded. Accordingly, patients were grouped according to their Kennedy classification into the first three classes only. These three groups facilitated recording specific results reflecting the QoL or OHRQoL with different posteriorly reduced and interrupted arches. The specific number of posterior occluding units was not reported, which is an important aspect that should be explored further, considering the body of evidence related to benefits of a functional dentition. ${ }^{11-33}$

The reactions and responses of patients in this study were somewhat at variance with what some of the literature has indicated. Whether this might in some way be on account of patients' lack of knowledge of the potentially negative effects of distal extension claspretained RPDPs for Kennedy Class I and II scenarios, including the risk they could pose to the remaining teeth, is difficult to say. 24,34 Research has shown that patients frequently do not use their distal extension clasp-retained RPDPs. ${ }^{1,21,42}$ A survey conducted by Jepson et al illustrates this point very well, with only $40 \%$ of RPDP patients actually wearing their dentures, and doing so especially when the anterior components were a priority. ${ }^{21}$

Perhaps also related to the observation of improved OIDP score after clasp-retained RPDP provision is the lack of knowledge related to the benefits of the non-interventional rationale of the SDA concept (which has indeed been accepted in South African Oral Health Policy) among undergraduate students, clinical teachers, and general practitioners. ${ }^{22,32}$ It follows that such a lack of awareness on the part of clinicians of the benefits that the SDA concept offers would likely not be conveyed to patients for whom such an option for managing reduced posterior occlusions is both viable and valid. ${ }^{22,32}$ It can also be speculated whether the fact that students' clinical education is premised upon achieving clinical requirements for graduation, and a "fee-for-service" dental care system compounds the problem of poor dissemination and uptake of the SDA concept. Thus, while the observed reduction in the total OIDP scores post-treatment indicates patients' satisfaction with prosthetic treatment that addressed their main complaint, whether this was so because it is actual or the perceived norm in clinical practice needs also to be investigated further.

In addition, the general absence among dental professionals of a patient-centered treatment approach has been noted. Gotfredsen and Walls were explicit about how patients have difficulties in voicing their opinions regarding oral function and their treatment requirements to practitioners. ${ }^{1}$ They suggest that patients be guided by evidence-based concepts when being treated to ensure a more patient-centered approach, and at the same time emphasizing the need for educating patients with regard to all treatment options, as well applying validated indicators to assess their needs. ${ }^{1}$ Knowledge related to the different oral health indicators introduced over time that address diverse aspects of QoL are thus very important. The OIDP index is a comprehensive indicator that addresses perceived needs of patients based on the daily activities of the individual. ${ }^{26,36,39}$ The severity of the condition with respect to function can be determined, and indeed, the changes following treatment with appropriately-designed RPDPs were very noticeable in the present

\section{http://repository.uwc.ac.za}


population. As a follow-up to this research, however, it would be useful to investigate the OHRQoL for patients with a classic SDA, and those with a reduced posterior occlusion but with acceptable numbers of posterior occluding pairs of teeth, while not having any interrupted arches and with intact anterior teeth. Such a design might unambiguously indicate whether the need for clasp-retained RPDPs, where cost is a major obstacle for readily obtaining these, is overstated in the South African context.

\section{Conclusions}

Considering the limitations of the current study, the following conclusions can be drawn:

1. In patients presenting with a range of posteriorly reduced, interrupted and/or discontinuous arches, the overall negative oral impacts were greatly reduced after provision of clasp-retained RPDPs.

2. Satisfaction with oral function was increased, and OHRQoL was improved across the three Kennedy groups.

3. Total OIDP score decreased significantly (from $20 \%$ to $5.9 \%$ ) subsequent to RPDP provision, as oral health status and level of satisfaction improved.

4. Whereas the value of RPDPs in this South African cohort, which is at variance with many global studies, was confirmed, the effects of other possible confounders to this apparent outcome need further study.

\section{Relevance of findings}

The findings of this study show the reliance on a clasp-retained RPDP by this cohort of partially dentate South African patients, where application of the SDA concept offering functional benefits could arguably have worked equally successfully. It is also apparent that a clinician-driven treatment approach is still used among the population studied, indicating an absence of patient-centeredness in treatment planning. Clinicians should not offer the RPDP treatment as the only treatment option, especially to patients who come from a low income and education group, when they present for treatment. In light of this, it can only be suggested that the outcomes of the study should be investigated further. 


\section{References}

1. Gotfredsen K, Walls AWG: What dentition assures oral function? Clin Oral Implants Res 2007;18:34-45

2. Sheiham A, Tsakos G: Oral health needs assessments. In: Pine CM, Harris R (eds): Community Oral Health, Chapter 4. Oxford, Wright Publishers, 2004

3. Zhang Q, Witter DJ, Bronkhorst EM, et al: Chewing ability in an urban and rural population over 40 years in Shandong Province, China. Clin Oral Investig 2013;17:1425-1435

4. Gerritsen AE, Allen FP, Witter DJ, et al: Tooth loss and oral health-related quality of life: a systematic review and meta-analysis. Health Qual Life Outcomes 2010;8:126-139

5. Elias EC, Sheiham A: The relationship between satisfaction with mouth and number and position of teeth. J Oral Rehabil 1998;25:649-661

6. Brennan DS, Spencer AJ, Roberts-Thomson KF: Tooth loss, chewing ability and quality of life. Qual Life Res 2008;17:227-235

7. Baba K, Igarashi Y, Nishiyama A, et al: The relationship between missing occlusal units and oral health-related quality of life in patients with shortened dental arches. Int $J$ Prosthodont 2008;21:72-74

8. Bradshaw JS: A taxonomy of social need. In McLachlan G (ed): Problems and Progress in Medical Care (ed 7). Oxford, Oxford University Press, 1972, pp.69-82

9. Narby B, Kronströ m M, Sö derfelt B, et al: Prosthodontics and the patient: Need becoming demand, demand becoming utilization. Int J Prosthodont 2007;20:183-189

10. Kä yser AF, Witter DJ, Spanauf AJ: Overtreatment with removable partial dentures in shortened dental arches. Aus Dent J 1987;32:178-182

11. Kä yser AF: The shortened dental arch: A therapeutic concept in reduced dentitions and certain high risk groups. Int J Perio Rest Dent 1989;9:426-449

12. Sarita PT, Witter DJ, Kreulen CM, et al: The shortened dental arch concept - attitudes of dentists in Tanzania. Community Dent Oral Epidimiol 2003;31:111-115

13. Witter DJ, van Elteren P, Kä yser AF: Signs and symptoms of mandibular dysfunction in shortened dental arches. J Oral Rehabil 1988;15:413-420

14. Witter DJ, van Elteren P, Kä yser AF, et al: Oral comfort in shortened dental arches. J Oral Rehabil 1990;17:137-143

15. Witter DJ, de Haan AF, Kä yser AF, et al: A 6-year follow-up study of oral function in shortened dental arches. Part I: Occlusal stability. J Oral Rehabil 1994;21:113-125

16. Kä yser AF: Shortened dental arches and oral function. J Oral Rehabil 1981;8:457-462 17. Kanno T, Carlsson GE: A review of the shortened dental arch concept focusing on the work by the Kä yser/ Nijmegen group. J Oral Rehabil 2006;33:850-862

18. Allen PF, Witter DF, Wilson NHF, et al: Shortened dental arch therapy: views of consultants in restorative dentistry in the United Kingdom. J Oral Rehabil 1996;23:481-485

19. Armellini DB, Von Fraunhofer JA: The shortened dental arch: A review of the literature. J Prosthet Dent 2004;92:531-535

20. De Sa e Frias V, Toothaker R, Wright RF: Shortened dental arch: A review of current treatment concepts. J Prosthodont 2004;13:104-110

21. Jepson NJA, Allen PF, Moynihan PJ, et al: Patient satisfaction following restoration of shortened mandibular dental arches in a randomized controlled trial. Int $\mathrm{J}$ Prosthodont 2003;16:409-414

\section{http://repository.uwc.ac.za}


22. Khan S, Chikte UME, Omar R: From classroom teaching to clinical practice: Experiences of senior dental students regarding the shortened dental arch concept. J Dent Educ 2014;78; 906-913

23. Korduner EK, Sö derfelt B, Kronströ m M, et al: Attitudes toward the shortened dental arch concept among Swedish general dental practitioners. Int J Prosthodont 2006;19:171-176

24. Owen CP: Appropriatech: Prosthodontics for the many, not just for the few. Int $J$ Prosthodont 2004;17:261-262

25. Sarita PT, Witter DJ, Kreulen CM, et al: Oral health status related to the shortened dental arch concept in Tanzania. Eur J Sciences 2007;115:265-274

26. Tan H, Peres KG, Peres MA: Do people with shortened dental arches have worse oral health-related quality of life than those with more natural teeth? A population-based study. Community Dent Oral Epidemiol 2015;43:33-46

27. Zeng X, Sheiham A, Tsakos G: Relationship between clinical dental status and eating difficulty in an old Chinese population. J Oral Rehabil 2008;35:37-44

28. Omar R: Reappraising prosthodontic treatment goals for older, partially dentate people: Part II. Case for a sustainable dentition? SADJ 2004;59:228-237

29. Allen PF, Witter DF, Wilson NHF: The role of the shortened dental arch concept in the management of reduced dentitions. Br Dent J 1995;179:355-357

30. Ueno M, Yanagisawa T, Shinada K, et al: Masticatory ability and functional tooth units in Japanese adults. J Oral Rehabil 2008;35:337-344

31.Fueki K, Yoshida E, Igarashi Y: A systematic review of prosthetic restoration in patients with shortened dental arches. Jap Dent Sci Rev 2011;47:167-174

32. Khan SB, Chikte UME, Omar R: Perceptions regarding the shortened dental arch among dental practitioners in the Western Cape Province, South Africa. SADJ 2012;67:60-68

33. Khan SB, Musekiwa A, Chikte UME, et al: Functional outcomes and relevance of prosthodontic interventions for shortened dental arches in adults: A Systematic Review. PloS One 2014;9:e101143.

34. Carlsson GE, Omar R: The future of complete dentures in oral rehabilitation. A critical review. J Oral Rehabil 2010;37:143-156

35. Slade GD, Spencer AJ: Development and evaluation of the Oral Health Impact Profile. Community Den Health 1994;11:3-11

36. Adulyanon S, Sheiham A: Oral impacts on daily performances. In Slade GD (ed): Measuring Oral Health and Quality of Life. Chapel Hill, UNC Press, 1997, pp. 151-160

37. Strassburger C, Heydecke G, Kerschbaum T. Influence of prosthetic and implant therapy on satisfaction and quality of life: A systematic literature review. Part ICharacteristics of the studies. Int J Prosthodont 2004;17:83-93

38. Strassburger C, Heydecke G, Kerschbaum T: Influence of implant and conventional prostheses on satisfaction and quality of life: A literature review. Part 2- Qualitative analysis and evaluation of the studies. Int J Prosthodont 2006;19:339-348

39. Hobdell M, Tsakos G, Ladrillo TE, et al: Using an oral health-related quality of life measure in three cultural settings. Int Dent J 2009;59:381-388

40. World Medical Organization. Declaration of Helsinki. Br Med J 1996;313:1448-1449

41. World Health Organization. A review of current recommendations for the organization and administration of Community Oral Health services in Northern and Western Europe. 
Copenhagen: WHO regional office for Europe. WHO Technical Report Series No. 826; 1982, p. 16-17

42. Frank RP, Brudvik JS, Le Roux B, et al: Relationship between the standards of removable partial denture construction, clinical acceptability and patient satisfaction. $J$ Prosthet Dent 2008;83:521-527 